\title{
Arbor
}

\section{Gestión clínica. Ventajas e inconvenientes}

\section{José Luis Temes Montes}

Arbor CLXX, 670 (Octubre 2001), 363-370 pp.

En la preocupación común de buscar una forma de participación efectiva de los profesionales y en la necesidad del cambio cultural que se requiere en las organizaciones a tal fin, para que garantice en todo momento el avance y consolidación de nuestro sistema sanitario, se analiza la gestión clínica como un posible marco de encuentro entre gestores y clínicos.

\section{Introducción}

Es de todos conocido que en la actualidad los Sistemas Sanitarios del mundo occidental están preocupados por el crecimiento de la denominada factura sanitaria, por los problemas de la universalización, por la accesibilidad y la equidad, por la libertad de elección, por la garantía de la calidad y por su eficiencia. El debate es creciente en todos los países, sin que haya respuestas sencillas. Las reformas y las contrareformas y los ensayos de nuevas fórmulas, son buena-prueba de ello.

Pero el problema que plantean estas reformas, impulsadas desde los ámbitos políticos y económicos, orientadas hacia la búsqueda de una organización sanitaria más flexible, basada en elementos de gestión empresarial y de competencia, es su implantación. Cualquier cambio 
debe ir acompañado de un cambio en la cultura de la organización y ello implica comprometer en él a actores con diferentes intereses.

(*) Este artículo es un resumen del capítulo del mismo título del libro «Gestión Clínica» J.L. Temes y B. Parra Mc Graw-Hil Interamericana 2.000.

Desde esta perspectiva, el desarrollo de la Gestión Clínica, se debe convertir en un elemento esencial para la mejora de la gestión de nuestro sistema sanitario. En un instrumento que permita adecuar la realidad de nuestro Sistema a las expectativas y posibilidades de la realidad de la sociedad española actual, y ésto se puede conseguir mediante la participación real de los profesionales en la gestión de los centros sanitarios y en la toma de decisiones, asumiendo sus propias responsabilidades, sin trasladarlas a otras instancias, y sin olvidar que cualquier éxito es un éxito de la organización a la que pertenecen.

\section{Análisis de la situación.}

La Llegada a la denominada Gestión Clínica a España se marca dentro de un contexto que podría esquematizarse en varios puntos.

$\left.1^{\circ}\right)$ Partimos de un sistema nacional de salud consolidado con un alto nivel técnico y profesional de calidad aunque con algunos problemas de desajuste, especialmente sensibles para los ciudadanos, como las listas de espera y algunos problemas de confort en los aspectos hosteleros. Este Sistema Nacional de Salud consolidado, tiene como componentes básicos como ya se ha expuesto en el primer capítulo, el aseguramiento universal, la financiación pública, la integración de políticas de promoción de la salud y de prevención de la enfermedad junto a prestaciones médicas y farmacéuticas, la equidad en el acceso y en la distribución de recursos y la descentralización.

$2^{\circ}$ ) La segunda característica en la que se enmarca la aparición de la Gestión Clínica en España es la elevada satisfacción de los usuarios con el actual sistema público reflejada en las encuestas que señalan reiteradamente que más del $70 \%$ de los ciudadanos está contento con el funcionamiento de la Sanidad Pública aunque también creen que son necesarios algunos cambios.

$3^{\circ}$ ) Que la Sanidad representa dentro de las áreas sociales la de mayor interés para los ciudadanos, tal y como se recoge en los barómetros sanitarios del Centro de Investigaciones Sociológicas (CIS). La sanidad representa el $32,8 \%$, la educación el $21,6 \%$, las pensiones el $16 \%$, la seguridad ciudadana el $11,8 \%$ y la defensa el $8 \%$.

$4^{\circ}$ ) El CIS también constata en su estudio sobre legitimidad institucional y grado de confianza en las instituciones políticas en el caso de Andalucía 
que la Corona es la primera, la Policía la segunda y el Servicio Andaluz de Salud la tercera Institución en legitimación y confianza.

$5^{\circ}$ ) Todos los sistemas sanitarios del mundo están hoy en crisis financiera.

Todo parece indicar que nos hallamos ante un sector que precisa reformas que mejoren algunos de los aspectos que demandan los ciudadanos al tiempo que moderen y racionalicen la utilización de los recursos financieros que los gobiernos destinan a los sistemas sanitarios. No se trata pues de profundas reformas que afecten al «núcleo duro» del sistema, sino reformas que perfeccionen un sistema consolidado.

Todos los Planes Estratégicos elaborados contemplan cuatro líneas comunes.

Parece difícil de discutir y sin duda debería de ser motivo de autocrítica de quienes hayamos tenido responsabilidad en ello, que el lenguaje de clínicos y gestores ha discurrido durante años por distintos caminos y en muchas ocasiones contrapuestos.

Parece claro que en los últimos tiempos y poco a poco se han producido algunos cambios importantes que han permitido una aproximación de clínicos y gestores. Así en las Administraciones Sanitarias ha calado la idea de que sin la complicidad de los profesionales es complicado, cuando no imposible, gestionar eficientemente los servicios sanitarios.

También es cierto que buena parte de los colectivos profesionales se han acercado en estos años a la gestión y son ya conscientes de que no es suficiente ser un buen médico y saben que sus posibilidades de desarrollo profesional están en buena medida vinculadas a su capacidad de complementar clínica y gestión.

No debemos olvidar que a lo largo de nuestra historia sanitaria más reciente y muy especialmente en las épocas de conflictos se ha formulado con reiteración el deseo de la participación de los profesionales en la gestión. Sin duda el transcurso del tiempo ha permitido la aproximación de criterios.

Si es cierto, como parece, que los gestores han llegado a la conclusión de que no podemos realizar nuestro trabajo sin los profesionales y que estos han llegado a la conclusión de que necesitan a los gestores y a la gestión para adaptarse a los tiempos que corren y seguir progresando, la pregunta es ¿Cómo hacerlo?

Hay algo que se ha enfocado incorrectamente a lo largo de años en distintos países, también en el nuestro, y es que los profesionales sanitarios y muy especialmente los médicos juegan un papel peculiar en la organización. Generan buena parte del gasto que pagan otros, lo hacen en muchos casos sin ser conscientes del coste de sus decisiones 
y además establecen unas relaciones muy particulares con sus «clientes», basadas en la extrema confianza de alguien que, en general, no está en una situación anímica normal. Los médicos además representan el más alto nivel científico de la organización en la materia y esto es un factor determinante en su relaciones con los demás trabajadores del hospital, que en muchos casos también son sus «clientes».

Es difícil pues que un colectivo así acepte de buen grado las decisiones sobre su trabajo provenientes de personas que no tienen o bien su formación o bien su desarrollo profesional.

Y si esto es así, como hoy parece claro, habrá que buscar fórmulas que permitan conciliar esa peculiaridad de los colectivos sanitarios y la necesidad de la gestión de recursos. En definitiva se ha de conseguir que la cadena de mando en un hospital no se fracture a nivel de los mandos intermedios, Jefes de Servicio, de las diversas unidades médicas. Para ello es preciso que como colectivo dejen la oposición y se sumen al proyecto convencidos de que las hostilidades han concluido y de que ellos, sólo ellos son quienes a nivel de servicio o unidad pueden gestionar su labor diaria.

Este camino nuevo, ya iniciado, precisa para su desarrollo reunir los siguientes requisitos:

$\left.1^{\circ}\right)$ Liderazgo: Para dirigir el proyecto, la Unidad Clínica de Gestión, es necesario una persona con liderazgo personal y profesional, «primun inter paris», con preparación técnica médica y gestora o posibilidad de adquirirla. $2^{\circ}$ ) Voluntariedad: Quienes se incorporen a este nuevo modelo organizativo deben de hacerlo de forma voluntaria, por lo menos en una primera fase de desarrollo del proyecto.

$3^{\circ}$ ) Transparencia: Tanto a la hora de exponer el proyecto como a la hora de elaborar los objetivos de lo que será su Contrato Programa. Estos objetivos habrán de ser claros, concretos, medibles y alcanzables. Transparencia también a la hora de adjudicar incentivos.

$\left.4^{\circ}\right)$ Singularidad: Aunque en algunos casos como en el de Hospital Clínico y Provincial de Barcelona se ha intentado abordar el nuevo esquema organizativo que requieren las Unidad de Gestión Clínica de una manera global desde el principio, en la mayoría de los hospitales se ha optado por comenzar con una o dos Unidades Clínicas muy seleccionadas, buscando líderes indiscutibles y limitando al máximo la posibilidad del fracaso.

En la corta experiencia española se ha podido ver como tras la puesta en marcha de alguna unidad en un hospital se ha desarrollado un clima primero de expectación que fue seguido por la solicitud de 


\section{Gestión clínica. Ventajas e inconvenientes}

información de otros servicios médicos tradicionales que empezaron a plantearse, a partir del ejemplo, la posibilidad de adoptar la nueva fórmula y lo han hecho a iniciativa de los propios profesionales.

El término GESTIÓN hace referencia al uso de los recursos. Mientras que LA CLINICA es la actividad médica directa al cuidado de los enfermos. Y así podríamos definir la «Gestión Clínica» como la utilización adecuada de los recursos para la mejor atención de los pacientes.

Esta definición implica que quienes toman días a día decisiones clínicas que comprometen los recursos económicos lo hagan en un marco de autoridad delegada y pactada con la Gerencia del hospital y que asuman la responsabilidad de sus propias decisiones.

\section{Principales ventajas e inconvenientes de la gestión clínica:}

\section{Ventajas:}

No precisa cambio normativo:

Quizá desde el punto de vista externo a las organizaciones sanitarias las principales ventajas de la Gestión Clínica frente otras fórmulas propuestas (Fundaciones, Empresas públicas, Institutos, etc) sería la de implantación interna, por lo que esta fórmula parece razonablemente ajena al debate parlamentario y por lo tanto al juego político entre Gobierno y oposición.

No concita rechazo político:

Podemos decir que frente a lo ocurrido con otras fórmulas de gestión, como las anteriormente mencionadas, la Gestión Clínica, al menos de forma frontal, no ha sufrido ese rechazo. Como veremos a lo largo de los capítulos, Administraciones de distinto signo político y de todas las tendencias han hecho una apuesta en mayor o menor medida por esta fórmula. Existen ya algunas experiencias en el INSALUD y en todas las Comunidades Autónomas con competencias sanitarias transferidas.

Paulatino y voluntario:

Se trata de llevar al Hospital cambios organizativos y culturales que permitan optimizar los recursos y mejorar la calidad de forma progresiva. 
Autonomía de gestión:

La Gestión Clínica provoca un desplazamiento formal de la toma de decisiones y así enlaza con una antigua reivindicación profesional $\mathrm{y}$ en muchos casos sindical de participar activa y constructivamente en las decisiones que se toman dentro del Hospital. Se establece un binomio imprescindible que habrá de realizarse en términos de resultados: Mejorar la eficacia, la eficiencia y la efectividad.

También agiliza la capacidad de respuesta de la organización: las Unidades de Gestión Clínica son más rápidas y más flexibles a la hora de tomar decisiones o de adaptarse a nuevas situaciones que el conjunto del Hospital.

Mejora las relaciones entre profesionales y usuario:

En general todas las Unidades se han marcado objetivos en esta línea y así las quejas, las encuestas post-hospitalización o las reclamaciones se han convertido en objetivos a obtener.

Mejora la motivación del personal:

La voluntariedad, la participación en la toma de decisiones y la firma responsable de Contratos Programas con la Gerencia de los Centros ha hecho que muchos profesionales abandonaran actitudes de desánimo ya que los objetivos o los resultados son pactados por ellos y dependen de ellos.

Mayor y mejor información:

La necesidad de tener que elaborar un Contrato Programa, de conocer los costes de los procesos que se realizan así como la actividad del conjunto de la Unidad Clínica, aporta una información de gran valor. El hecho de conocer esta información estimula a los grupos así constituidos a proponer líneas de actuación y objetivos de mejora.

\section{Inconvenientes:}

Presión sindical:

En el hospital «Reina Sofía» esta presión se produjo en el momento de la implantación de la primera Unidad de Gestión Clínica, la de 
Aparato Digestivo y aunque probablemente haya habido razones de carácter local no extrapolables a otros ámbitos, se produjo una campaña a través de los medios de comunicación tratando de llevar a la opinión pública la idea de que se estaba realizando una privatización encubierta de una parte de los Servicios Asistenciales. A pesar de la falta de rigor de este argumento ya que no hay nada tan alejado del debate público-privado en sanidad como la Gestión Clínica, un grupo de organizaciones sindicales constituyó una plataforma contra la privatización de los servicios médicos que tuvo escasa repercusión dentro del Hospital, aunque si en la prensa. Poco tiempo después esta presión sindical desapareció.

Tensión entre los grupos profesionales:

En nuestro caso un grupo de cirujanos de Aparato Digestivo rechazaron la oferta inicial de integrarse en la Unidad de Gestión Clínica. Pasados unos meses reivindicaban ante la gerencia y ante la Junta Facultativa su derecho a formar parte de ella. Entre una y otra posición medió la puesta en marcha de la citada Unidad y el desarrollo de unas expectactivas distintas de los profesionales que se habían incluido en ellas.

Críticas de desestructuración organizacional de los servicios:

Ha habido quienes han cuestionado la ruptura de los servicios clásicos con la aparición dentro del Hospital de dos tipos de Servicios, los integrados en la nueva fórmulas y los tradicionales. Parece difícil rebatir que esto sea así pero también parece cierto que históricamente en todos los Hospitales ha habido Servicios más eficientes que otros y son estos últimos los que muestran una mayor disposición a introducir esta nueva fórmula de gestión en sus servicios.

En resumen y haciendo balance entre los inconvenientes y las ventajas nuestra apuesta es decididamente a favor de la creación de Unidades de Gestión Clínica, a la incentivación de este modelo y a su implantación progresiva en los Hospitales.

\section{Bibliografía}

1 T. White. Gestión Clínica. Masson. 1.997.

2 DHSS. NHS management inquiry. London: HMSO. 1983.

3 DHss. Working for Patiens. London: HMSO. 1989. 


\section{José Luis Temes Montes}

370

4 Heyssell RM, Gaintner JR, KUEs IW, Jones AA, LipStein SH. Decentralised management in a teaching hospital. N Engl J Med 1984: 310 (22): 1477-80.

5 Williams A. Medical ethics: health service efficiency and clinical freedom. Nuffield/York Portfolio No. 2 London: Nuffield Provincial Hospitals Trust. 1985. 